\title{
Time course of histopathological changes after bleomycin sclerotherapy in rabbit gallbladders as a model for simple hepatic cysts
}

\author{
$\mathrm{LONG} \mathrm{LI}^{1 *}$, YONG-HAO LAO ${ }^{1 *}$ and NAN ZHANG ${ }^{2}$ \\ ${ }^{1}$ Division of Interventional Radiology, Department of Medical Imaging, Guangdong Provincial Corps Hospital \\ of Chinese People's Armed Police Forces, Guangzhou Medical University; ${ }^{2}$ Department of Pathology, \\ Liwan Central Hospital of Guangzhou City, Guangzhou, Guangdong 510507, P.R. China
}

Received April 17, 2021; Accepted July 1, 2021

DOI: $10.3892 /$ br.2021.1451

\begin{abstract}
Bleomycin sclerotherapy is used in the treatment of cystic lesions; however, the histopathological changes are undefined. Present animal models of cystic diseases are not adequate for the study of sclerotherapy of hepatic cysts, primarily because the established cysts in these models are too small in size. The aim of the present study was to establish a new animal model of simple hepatic cysts, and assess the histopathological changes after bleomycin sclerotherapy. Rabbit gallbladder, with ligaturing of the cholecystic duct whilst preserving cholecystic vessels, was used as a model for simple hepatic cysts. Bleomycin $(2 \mathrm{mg}$ dissolved in $1 \mathrm{ml}$ saline) was injected into the aspirated gallbladder, gallbladder tissue was harvested (after 1, 7, 14, 28, 42, 56 and 84 days) and histopathological changes were evaluated ( $n=4$ per group). Additionally, control rabbit gallbladders were injected with $1 \mathrm{ml}$ saline and sampled after 14 days $(n=4)$. Histopathological changes were evaluated using hematoxylin-eosin and Masson's trichrome staining, and immunohistochemistry for CD20-, CD43- and CD68-positive cells was performed. The integrated optical density (IOD) of immunohistochemical staining and average positive stained area percentage (APSAP) of collagen were quantitatively analyzed. The results revealed gallbladders in the control group had regular epithelial cells
\end{abstract}

Correspondence to: Dr Long Li, Division of Interventional Radiology, Department of Medical Imaging, Guangdong Provincial Corps Hospital of Chinese People's Armed Police Forces, Guangzhou Medical University, 268 Yanling Road, Guangzhou, Guangdong 510507, P.R. China

E-mail: radiolilong@hotmail.com

*Contributed equally

Abbreviations: APSAP, average positive stained area percentage; $\mathrm{HE}$, hematoxylin eosin; IOD, integral optical density

Key words: bleomycin, sclerotherapy, simple hepatic cyst, pathology with no visible inflammation or fibrosis. In the experimental group, epithelial cells were swollen and necrotic on the first day, and were replaced gradually by single-layer flat cells from day 56. Inflammatory infiltration was found in the submucosa, and the IOD of T cells, B cells and macrophages were highest on day 1 , and these parameters declined gradually, eventually disappearing. The APSAP of collagen was highest on day 7 , and gradually declined thereafter. The results suggest that histopathological changes after bleomycin sclerotherapy of a simple hepatic cyst model were characterized by sequential epithelial destruction, inflammatory cell infiltration, collagen proliferation and epithelial partial regeneration.

\section{Introduction}

Percutaneous aspiration with sclerotherapy is a minimally invasive, simple, safe, inexpensive and reasonably effective treatment for cystic diseases, such as simple hepatic, renal, splenic, pancreatic and ovarian cysts, as well as for lymphoceles (1-4). Bleomycin has been used successfully for management of cysts $(3,4)$; it is an antibiotic chemotherapeutic agent derived from Streptomyces verticillus, which was first used as a sclerosant for malignant pleural effusions in 1976 (5). Intralesional bleomycin injection has been used successfully for the treatment of cystic diseases, including lymphoceles since 1977 (6), cystic craniopharyngiomas since 1985 (7), bronchogenic cysts since 1992 (8), simple renal cysts since 2012 (9) and simple hepatic cysts since 2015 (10). Advantages of percutaneous bleomycin sclerotherapy for treatment of cystic diseases are minimal inflammatory reactions, modest cost as only a single treatment session is required, good tolerance as it causes little pain and no pulmonary toxicity when performed with the proper precautions (3,9-11).

The sclerosing mechanisms of intracyst bleomycin injection are not understood. For example, they may be similar to those of pulmonary fibrosis induced by intratracheal bleomycin instillation (12); however, that inference has not been confirmed by histopathological examination, and there are no animal models for evaluation of percutaneous sclerotherapy of hepatic cystic disease. Rodent models of drug-induced polycystic diseases (including liver, kidney and ovary) have been 
widely used, but they have limitations. For example, their pathogenesis is different from that of simple cysts, their modeling time is long and they have multiple small cysts (13-15). Thus, such animal models are not suitable for studying percutaneous sclerotherapy of cysts. The animal model of simple ovarian cysts, established by unilateral total salpingectomy, has been used successfully to evaluate the effectiveness and safety of aspiration sclerotherapy, but only $70 \%$ of experimental animals formed macroscopic ovarian cysts of $\sim 10 \mathrm{~mm}$ (16). Therefore, in the present study, a new animal model of a simple hepatic cyst was established, and the chronological histopathological changes after intracyst bleomycin injection was assessed.

\section{Materials and methods}

Ethical considerations. The present study was approved by the Biomedical Ethics Committee of Animal Experiments of Guangdong Medical Laboratory Animal Center (Guangdong, China; approval no. B201610-5). An Accreditation Certificate from the China National Accreditation Service for Conformity Assessment has been granted to this facility. All experimental procedures were performed in accordance with the Guide for the Care and Use of Laboratory Animals (National Institutes of Health) (17). Every effort was made to minimize animal suffering and to use only the number of animals necessary for the acquisition of reliable data.

Animal model. A total of 32 New Zealand rabbits of either sex (11 males, 21 females), weighing $2.6 \mathrm{~kg}$ (range, 2-3 kg) at the beginning of the study, were obtained from Guangdong Medical Laboratory Animal Center, China (order no. 44411600002998). Rabbits were housed in separate cages in the animal experimental center, with an ambient temperature of $18-24^{\circ} \mathrm{C}$ and a $12 \mathrm{~h}$ light/dark cycle. Rabbits were provided ad libitum access to food and water during the experimental period. Rabbits were anesthetized by injection of $30 \mathrm{mg} / \mathrm{kg}$ sodium pentobarbital in the ear vein (Shanghai Chemical Reagent Company, China National Pharmaceutical Group Corporation) and intramuscular injection of $5 \mathrm{mg} / \mathrm{kg}$ xylazine hydrochloride (Shanghai Chemical Reagent Company, China National Pharmaceutical Group Corporation) after $12 \mathrm{~h}$ of fasting. By means of surgical laparotomy, the cholecystic duct was ligated with a 3/0 surgical suture, whereas cystic vessels were retained to ensure good blood supply and to avoid ischemic necrosis (Fig. 1). Direct gallbladder puncture was performed as far as possible from gallbladder vessels using a 22-gauge butterfly needle. Bile was aspirated, and the gallbladder was irrigated 3-4 times with saline until clear. The surgical time ran between 45 and $60 \mathrm{~min}$. Anesthesia with a standard dose was well tolerated.

Experimental design. The rabbits were randomly divided into a control group and seven experimental groups according to different time points after bleomycin sclerotherapy $(1,7,14$, $28,42,56$ and 84 days; $n=4$ per group). In animals in the experimental group, after gallbladder content was aspirated as thoroughly as possible, $2 \mathrm{mg}$ dissolved in $1 \mathrm{ml}$ saline bleomycin hydrochloride (Takasaki Plant; Nippon Kayaku Co., Ltd.) was injected and allowed to remain in the evacuated gallbladder until the experiment ended. For animals in the control group, $1 \mathrm{ml}$ normal saline was injected, which remained in the

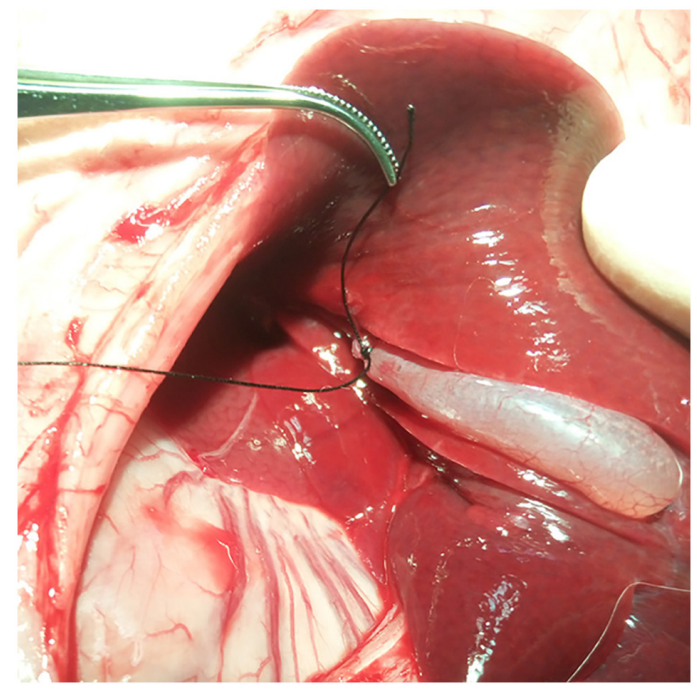

Figure 1. Animal model of simple hepatic cysts. The cholecystic duct was ligated, with preservation of cholecystic vessels. The gallbladder was then irrigated with normal saline until the cystic fluid became clear.

evacuated gallbladder. Rabbits received penicillin 10 million units/kg per day intramuscularly for three consecutive days after completion of the procedure, and were sacrificed at the end of the experimental period. At 1, 7, 14, 28, 56 and 84 days after the bleomycin injection, animals in experimental groups were euthanized by intravenous injection of $150 \mathrm{mg} / \mathrm{kg}$ potassium chloride (Shanghai Chemical Reagent Company; China National Pharmaceutical Group Corporation) under general anesthesia induced by intravenous injection of $30 \mathrm{mg} / \mathrm{kg}$ pentobarbital sodium via the marginal ear vein; animals in the control group were euthanized at day 14. The gallbladders and the surrounding liver parenchyma were resected and evaluated histologically and immunohistochemically.

Histopathological processing. The cholecystic tissue specimens were fixed in $4 \%$ paraformaldehyde for $24 \mathrm{~h}$ at room temperature, trimmed appropriately, dehydrated in graded series of alcohol solutions and embedded in paraffin according to standard procedures (18). Paraffin sections of $4 \mu \mathrm{m}$ thickness were cut and mounted on glass microscope slides.

Hematoxylin and eosin (H\&E) staining was performed using a H\&E Staining kit (cat. no. G1005; Wuhan Servicebio Technology Co., Ltd.), as described previously (14). Masson's trichrome staining was performed using a Masson's Trichrome Stain kit (cat. no. G1006; Wuhan Servicebio Technology Co., Ltd.) as described previously (19).

Infiltration by T-lymphocytes, B-lymphocytes and macrophages into the tissue was detected immunohistochemically using a panel of monoclonal antibodies against CD20, CD43 and CD68 (20). Immunohistochemical staining was performed using the Streptavidin-Biotin-Complex method, according to the Sixth Edition of Dako's Educational Guidebook to Immunohistochemical Staining Methods (21). Sections were deparaffinized in xylene $(2 \times 10 \mathrm{~min})$ and rehydrated using an alcohol gradient: $100 \%$ ethanol (2x10 min), 95\% ethanol (1x8 min), $80 \%$ ethanol (1x5 min) and $70 \%$ ethanol (1x5 min), followed double-distilled H2O (1x10 min). Nonspecific background staining was blocked using Background Sniper (cat. 

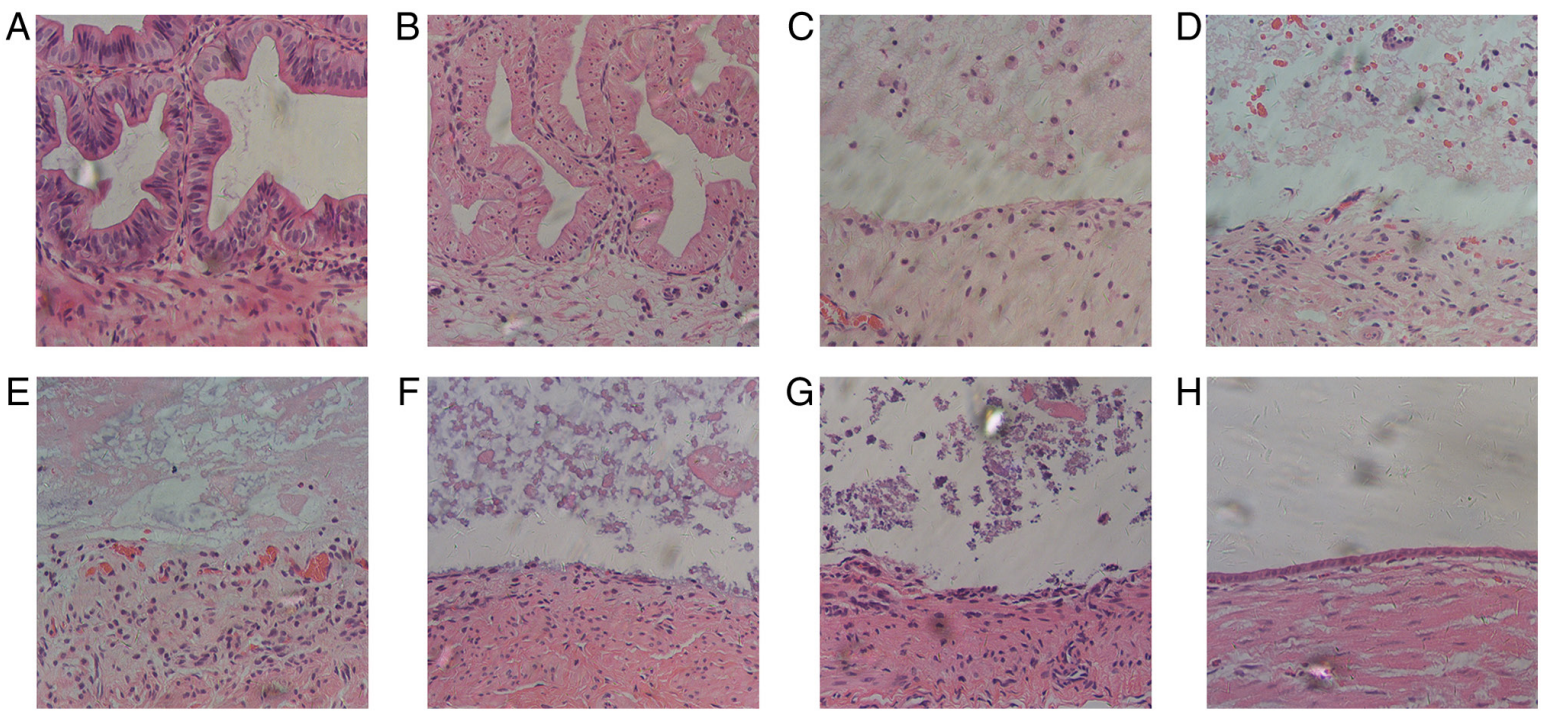

Figure 2. Time course of cyst epithelial changes after bleomycin sclerotherapy in the rabbit model of simple hepatic cysts (hematoxylin and eosin staining; magnification, x200). (A) In the control group, the integrity of the mucosal structure and the epithelial cells is visibly maintained. (B) A total of 1 day after bleomycin sclerotherapy, the epithelial cells had ballooned and degenerated. (C) At 7 days after bleomycin sclerotherapy, the mucosa was visibly disrupted. (D-H) From days 14-84, the mucosal structures were absent, and edema of the lamina propria edema gradually decreased and disappeared. (G) At 56 days after bleomycin sclerotherapy, a single layer of scattered flat cells was present. (H) At 84 days, the gallbladder mucosa had been replaced by a single layer of flat epithelial cells.

no. BS966M; Biocare Medical, LLC), 3\% BSA (Sigma-Aldrich; Merck KGaA) in PBS, and 5\% normal goat-serum solution (cat. no. SP-9000; OriGene Technologies, Inc.) for $30 \mathrm{~min}$ at room temperature. Slides were incubated overnight at $4^{\circ} \mathrm{C}$ for $1 \mathrm{~h}$ at room temperature with primary antibodies in $3 \%$ BSA-PBS. Primary antibodies used were anti-CD20 (1:3,000; cat. no. GB11281; Beijing Solarbio Science \& Technology Co., Ltd.), anti-CD43 (1:1,500; cat. no. GB11066; Beijing Solarbio Science \& Technology Co., Ltd.) and anti-CD68 (1:1,500; cat. no. GB11067; Beijing Solarbio Science \& Technology Co., Ltd.). After washing five times with 0.05\% Brij-35 (Abmole Bioscience, Inc.) in PBS for 1 min, slides were incubated with horseradish peroxidase-conjugated goat anti-rabbit/mouse IgG secondary antibody at 1:1,000 dilution (cat. no. K5007; Dako; Agilent Technologies $\mathrm{GmbH}$ ) for $1 \mathrm{~h}$ at room temperature. The criterion for positive staining with the CD20, CD48 and CD68 antibodies was the presence of pale brown particles in cell nuclei.

Microscope slides were examined by a pathologist with $>15$ years of working experience, and who was blinded to the experimental conditions. At a magnification of x200, two sections in five random fields of view in each region of interest were selected for further analysis. Images were taken using a Nikon Eclipse Ti inverted fluorescence microscope (Nikon Corporation) equipped with the Nikon NIS-Elements imaging analysis software version 4.60.00. The average positive stained area percentage (APSAP) of collagen fiber revealed by Masson's trichrome staining was quantitatively analyzed using Image-Pro Plus version 7.0 (Media Cybernetics, Inc.) (22). The immunohistochemical images with integrated optical density (IOD) of positive expression, which reflected the dynamic changes and distribution characteristics of $\mathrm{T}$ cells, $B$ cells and macrophages in gallbladder tissues, were quantitatively analyzed using Image-Pro Plus (23).

Statistical analysis. Data are presented as the mean \pm standard error of the mean. Statistical comparisons of the intensities of immunohistochemical staining and Masson's trichrome staining were performed using a one-way ANOVA followed by a post-hoc Tukey's test. All statistical analyses were performed using SPSS version 20 (IBM, Corp.) Graphs were generated using GraphPad Prism version 6 (GraphPad Software, Inc.). P $<0.05$ was considered to indicate a statistically significant difference.

\section{Results}

Histopathological evaluation of the rabbit model of simple hepatic cysts. All rabbits in the control group survived after the simple hepatic cyst model was established, with normal appetite, activity and excretion. A total of 14 days after intracyst saline injection, autopsies revealed an intact structure and clear boundary between the gallbladder and hepatic capsule. H\&E staining demonstrated that epithelial cells of the gallbladder were arranged neatly with prominent nucleoli and abundant cytoplasm, and the mucosa plica were flourishing without morphological changes (Fig. 2A). Immunohistochemical staining of CD20, CD43 and CD68 demonstrated no inflammatory-cell infiltration in the cyst wall, whether by B-lymphocytes 2 (Fig. 3A), T-lymphocytes (Fig. 4A) or macrophages (Fig. 5A). Masson staining revealed no collagenous fibers in the mucous layer, and the basal side of epithelial cells was fixed tightly by a small amount of fibrin; similarly, collagen fibers in the lamina propria mucosae and submucous layer were lined up tightly (Fig. 6A).

Time course of cyst epithelial changes after bleomycin sclerotherapy in the rabbit model of simple hepatic cysts. All 28 rabbits in the experimental group survived the entire duration of the experimental procedure, with normal behavior, mental state and body weight, and no complications were observed.

The first day after bleomycin sclerotherapy, the epithelial cells were swollen and had balloon-like degeneration; the nuclei were pyknotic and deformed, appearing as small circles. 

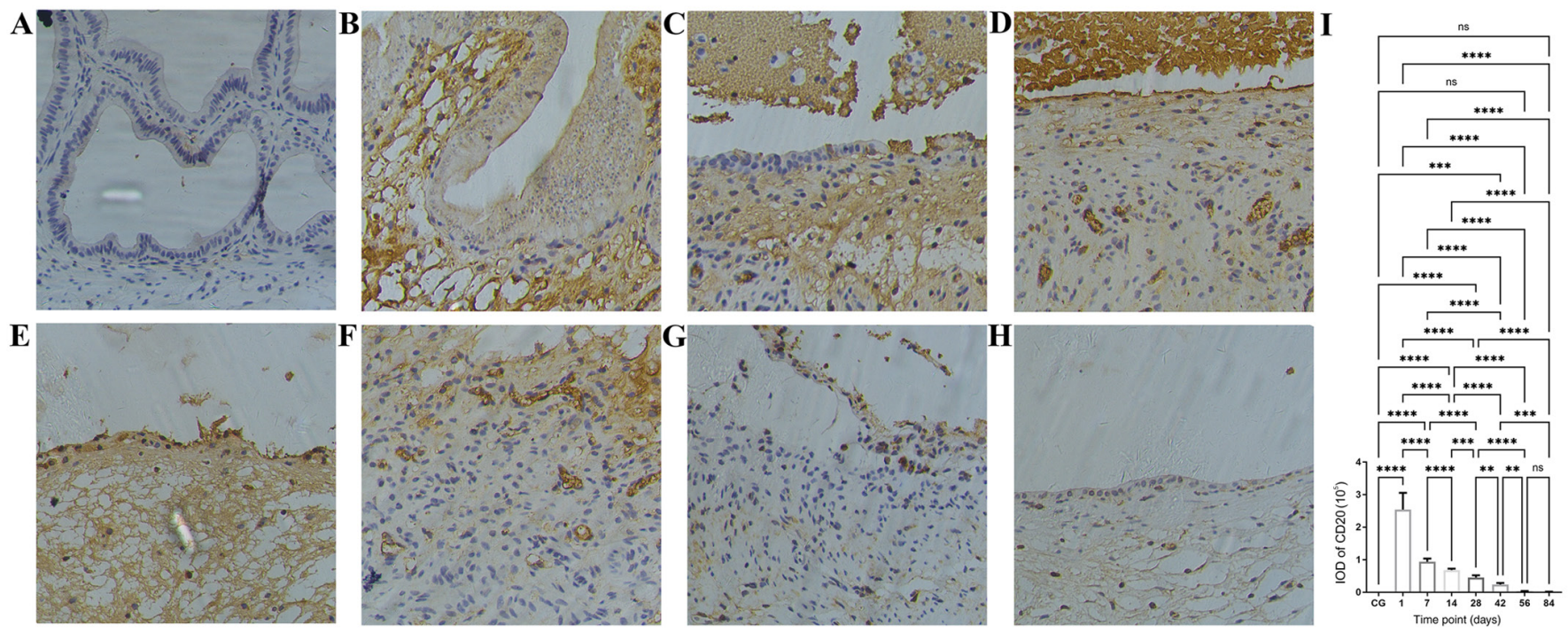

Figure 3. Time course of B-lymphocyte infiltration following bleomycin sclerotherapy in the rabbit model of simple hepatic cysts based on anti-CD20 antibody immunohistochemical staining. (A) In the control group, there were no B-lymphocytes in the cyst wall. (B) Following intracyst bleomycin injection, after 1 day, B-lymphocytes had extensively infiltrated the submucosal layer and lamina propria. (C and D) At 7 and 14 days after intracyst bleomycin injection, B-lymphocytes extensively infiltrated the submucosal layer, lamina propria, and the destroyed mucosal areas. (E-H) At 28 and 84 days after intracyst bleomycin injection, B-lymphocyte infiltration decreased but persisted throughout the experiment. (I) Quantitative analysis of IODs of anti-CD20 immunohistochemical staining at the different time-points. Detailed data and statistics to produce this graph are listed in Table SI. Magnification, $\mathrm{x} 200 .{ }^{* * *} \mathrm{P}<0.01,{ }^{* * * *} \mathrm{P}<0.001$, ${ }^{* * * *} \mathrm{P}<0.0001$. IOD, integrated optical density; CG, control group.
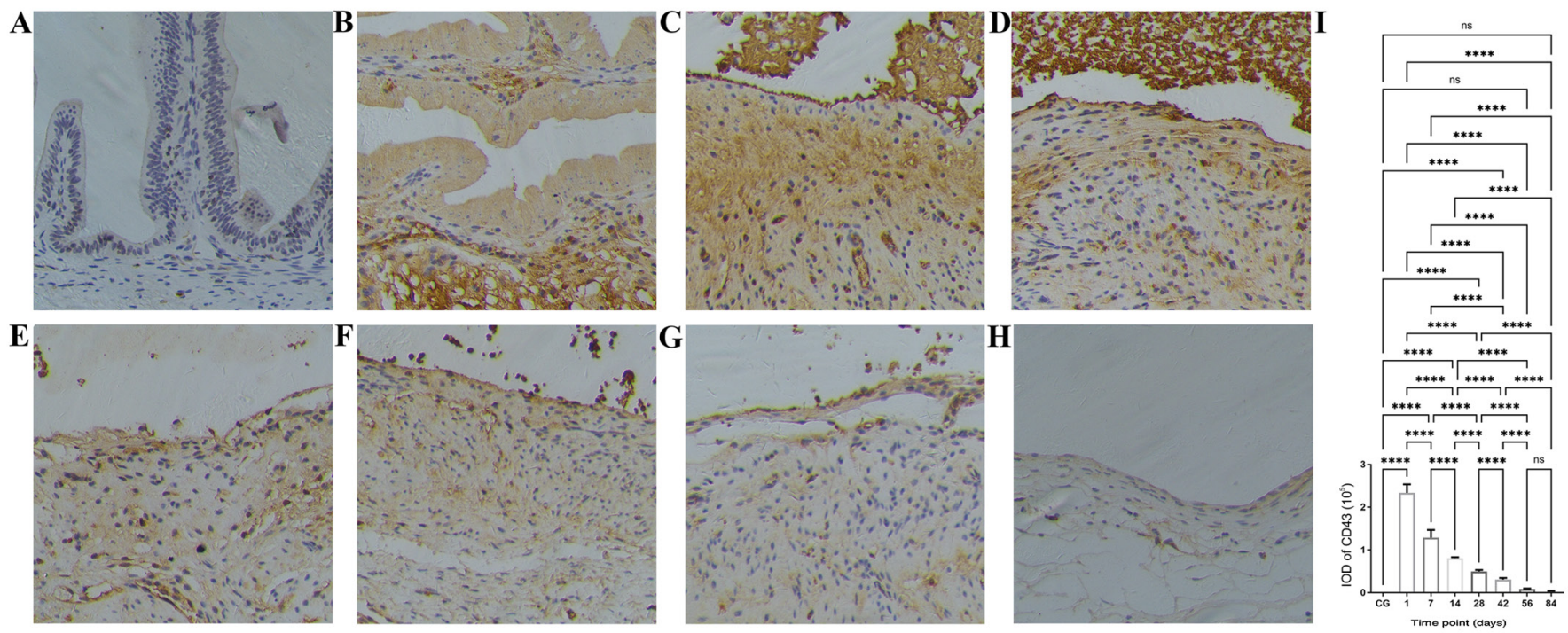

Figure 4. Time course of T-lymphocyte infiltration following bleomycin sclerotherapy in the rabbit model of simple hepatic cysts based on anti-CD43 antibody immunohistochemical staining. (A) In the control group, T lymphocytes were not present in the cyst wall. (B) At 1 day after intracyst bleomycin injection, T-lymphocytes extensively infiltrated the submucosal layer and lamina propria. (C and D) At 7 and 14 days after intracyst bleomycin injection, T-lymphocytes extensively infiltrated the submucosal layer, the lamina propria and the destroyed mucosal areas. (E-H) At 28 and 84 days after intracyst bleomycin injection, T-lymphocyte infiltration gradually decreased with time, but persisted during the experiment. (I) Quantitative analysis of IODs of anti-CD43 immunohisto-

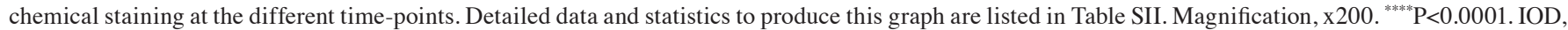
integrated optical density; CG, control group.

The lamina propria was edematous, with a few neutrophils and fibroblasts present (Fig. 2B).

A total of 7 days after bleomycin sclerotherapy, the mucosal structures were destroyed and had disappeared, and the lamina propria was detached by the mixed cellular infiltrate of fibroblasts, collagen fibers and inflammatory cells (Fig. 2C).

From days 14-84 following bleomycin sclerotherapy, the lamina propria edema decreased gradually and disappeared, and fibrosis and inflammatory response continually decreased (Fig. 2D-H). At 56 days, the mucous layer contained irregularly flattened epithelial cells (Fig. 2G), which were replaced almost completely at 84 days (Fig. $2 \mathrm{H}$ ).

Time course of inflammatory cell infiltration after bleomycin sclerotherapy in the rabbit model of simple hepatic cysts. The time course in the IODs of inflammatory cells in 

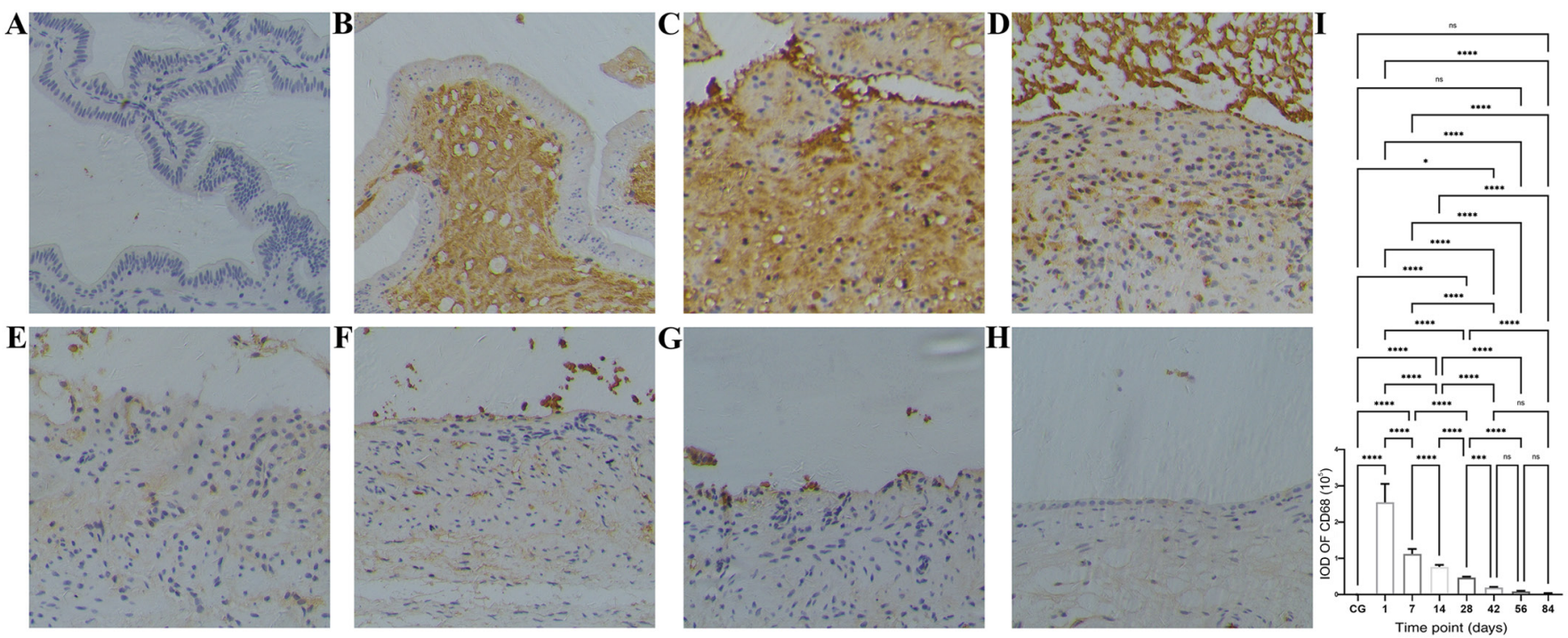

Figure 5. Time course of macrophage infiltration after bleomycin sclerotherapy in the rabbit model of simple hepatic cysts based on anti-CD68 antibody immunohistochemical staining. (A) Control group, no macrophages were present in the gallbladder wall. (B) At 1 day after intracyst bleomycin injection, macrophages extensively infiltrated the submucosal layer and lamina propria. (C and D) At 7 and 14 days after intracyst bleomycin injection, macrophages extensively infiltrated the submucosal layer, lamina propria and the destroyed mucosal areas. (E-H) At 28 and 84 days after intracyst bleomycin injection, macrophages gradually decreased, but exhibited a persistent presence throughout the duration of the experiment. (I) Quantitative analysis of IODs of anti-CD68 immunohistochemical staining at the different time-points. Detailed data and statistics to produce this graph are listed in Table SIII. Magnification, x200. ${ }^{*} \mathrm{P}<0.05,{ }^{* * *} \mathrm{P}<0.001,{ }^{* * * * *} \mathrm{P}<0.0001$. IOD, integrated optical density; CG, control group.
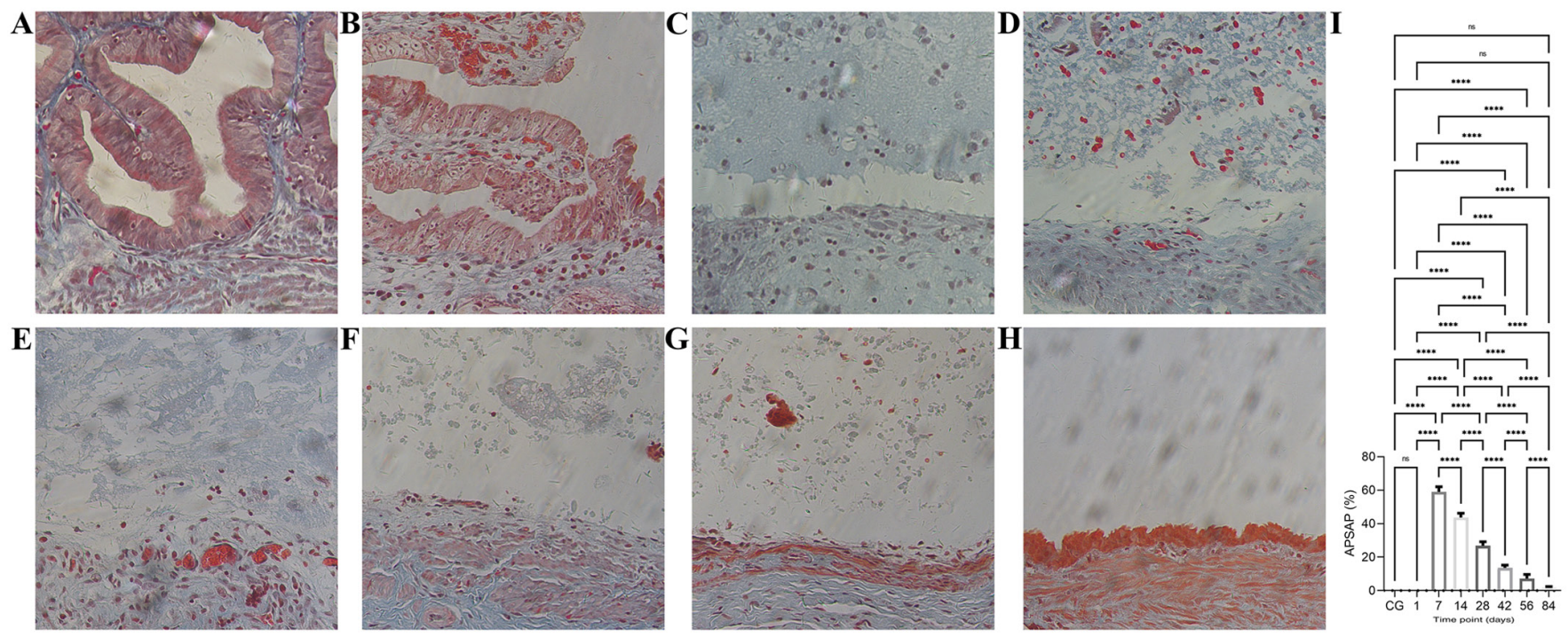

Figure 6. Time course of collagen proliferation following bleomycin sclerotherapy in the rabbit model of simple hepatic cysts based on Masson's trichrome staining.. Collagen is stained blue. (A) In the control group, collagenous fibers were not present in the mucous layer. (B) At 1 day after intracyst bleomycin injection, few fibers were present in the submucosa. (C) At 7 days after intracyst bleomycin injection, blue-stained collagen fibers filled the field of view in the destroyed mucosal areas. (D-H) From days 14-84 after intracyst bleomycin injection, the blue-stained collagen fibers gradually decreased and almost disappeared. (I) Quantitative analysis of APSAPs of collagen fibers of Masson's trichrome staining at different time-points. Detailed data and statistics to produce this graph are listed in Table SIV. Magnification, $\mathrm{x} 200 .{ }^{* * * * *} \mathrm{P}<0.0001$. APSAP, average positive stained area percentage; CG, control group.

the cyst wall at various time points after intracyst bleomycin injection is shown in Figs. 3-5 and Tables SI-III. The number of B cells (CD20-positive, Fig. 3I), T cells (CD43-positive, Fig. 4I) and macrophages (CD68-positive, Fig. 5I) peaked at the first day after intracyst bleomycin injection, and then gradually declined, with each day's values lower than the preceding day's values $(\mathrm{P}<0.01)$. IODs at 84 days after bleomycin were still significantly higher than in the control group $(\mathrm{P}<0.01)$.
One day after intracyst bleomycin injection, inflammatory cells (B-lymphocytes, T-lymphocytes and macrophages) were extensively present in the submucosal layer and lamina propria (Fig. 3B, 4B and 5B). The IODs of stained inflammatory cells were significantly higher after 1 day than at other time-points (Fig. 3I, 4I and 5I; P<0.01). A total of 7 and 14 days after bleomycin injection, inflammatory-cell infiltration was present in the submucosal layer and lamina propria as well as in the destroyed mucosal areas (Figs. 3C and D, 4C-D and 5C-D). 
From 28 to 84 days after intracyst bleomycin injection, inflammatory cell infiltration in the submucosal layer decreased gradually, but was present throughout the experiment (Figs. 3E-H, 4E-H and 5E-H).

Time course of collagen proliferation after bleomycin sclerotherapy in the rabbit model of simple hepatic cysts. The time course of the APSAP of collagen fibers stained with Masson's trichrome in the cyst wall at the various time points after intracyst bleomycin injection is given in Fig. 6 and Table SIV. Maximum staining was recorded on day 7 , which gradually decreased and almost disappeared by day 84 (Fig. 6I, $\mathrm{P}<0.01$ ). At 1 day after intracyst bleomycin injection, a few new collagen fibers were present in the submucosal layer (Fig. 6B). At 7 days, abundant collagen fibers were evident amidst destroyed mucosal areas (Fig. 6C), and the APSAP was significantly increased compared with that of day 1 (Fig. 6I, $\mathrm{P}<0.01$ ). From days $14-84$ after intracyst bleomycin injection, staining of collagen fibers decreased gradually and almost disappeared (Fig. 6D-H).

\section{Discussion}

The results of the present study indicate that the time course of histopathological changes in the rabbit gallbladder as a model for studying bleomycin sclerotherapy of simple hepatic cysts can be divided into four phases: i) The epithelial destruction phase, occurring within 14 days; ii) the inflammatory infiltration phase, peaking the first day after bleomycin sclerotherapy and persisting throughout the experiment; iii) the collagenous proliferation phase, demonstrated at day 7 after bleomycin sclerotherapy, then gradually decreasing and eventually disappearing; and iv) the epithelial regeneration phase of a single layer of flat cells from 56 days after bleomycin sclerotherapy.

To the best of our knowledge, the animal model of a simple hepatic cyst described in the present study is the only available study on creating such a model in the English literature. Simple hepatic cysts consist histologically of a single layer of cuboidal or flattened epithelium, which is derived from developmental anomalies of biliary epithelium, and arise from misplaced or detached biliary anlage (24). These features are the theoretical basis for the animal model of this study. Furthermore, the anatomical site of the rabbit gallbladder is convenient for making surgical procedures and is independent of the digestive system, such that its function and survival are not affected after operation. The practical basis of the rabbit gallbladder model is derived from animal experiments of chemical ablation of the gallbladder, with various sclerosants that emerged from the late 1980's to the early 2000's (25-27). The results of the present study suggest that the animal model of a simple hepatic cyst established by ligating the cholecystic duct and preserving cholecystic vessels is more reliable, operable and repeatable than other reported models of cystic diseases.

Destruction of the epithelial lining of the inner surface of the cyst wall to prevent intracystic fluid secretion is the goal of percutaneous aspiration and sclerotherapy for cystic diseases (1-4). Studies using experimental animal models of bleomycin-induced pulmonary fibrosis have confirmed that the destruction of the alveolar epithelium is an initiating event in the pathogenesis of lung fibrosis and in acute lung injury (28).
Bleomycin, in the presence of iron and oxygen, can generate reactive oxygen species and damage DNA by causing singleand double-strand breaks, a mechanism thought to be the basis for its antitumor activity and presumed mechanism of cytotoxicity and tissue injury (29). However, the doses used in the rodent model of fibrosis do not appear to cause significant DNA damage (12), thus making it unclear if this is the basis for of lung fibrosis induced by bleomycin $(12,28)$. A bleomycin concentration of $2 \mathrm{mg} / \mathrm{ml}$ used in the present study is based on the experimental model of bleomycin-induced lung and skin fibrosis $(29,30)$. The total amount of bleomycin saline solution injected into the cyst cavity $(1 \mathrm{ml})$ is sufficient to fill the rabbit gallbladder, based on the amount of cyst fluid aspirated initially. The epithelial destruction resulting from this bleomycin dose appeared slightly earlier than that of bleomycin-induced lung injury in animals; that is, epithelial degeneration was seen on the first day, and the mucosa disappeared within 7 days, whereas alveolar epithelial cell injury and death occurred 7-14 days after intratracheal bleomycin instillation (28). Whether this difference is caused by the organ specificity of bleomycin hydrolase, which is necessary to inactivate the bleomycin $(12,28)$, is unknown.

Bleomycin-induced inflammation in animal models of pulmonary fibrosis is initiated in response to epithelial-cell damage and release of pro-inflammatory mediators, which results in enhanced vascular permeability for the recruitment of leukocytes $(28,31)$. This process also results in the recruitment and activation of immune cells, including macrophages and lymphocytes, that produce cytokines and chemokines (32). In the present study, infiltration of the gallbladder wall with T-lymphocytes, B-lymphocytes and macrophages suggested that similar inflammatory activities occurred. In clinical practice, several minor complications after percutaneous bleomycin sclerotherapy for treatment of cystic diseases can be encountered, including transient low-grade fever, local skin ulceration, local swelling and redness at the site of injection $(3,7,9,33)$, which may be the result of bleomycin-induced inflammation. This prolonged inflammatory response may explain the clinical phenomenon in which cysts refill partially in the initial stages following bleomycin sclerotherapy and regress gradually thereafter, and even disappear completely $(9,10)$. Therefore, the optimal follow-up time for assessing the therapeutic outcome should be after resolution of inflammation, and this should be no less than 12 weeks (84 days) after intracyst bleomycin injection.

Tissue fibrosis followed by cyst shrinking is the treatment outcome of percutaneous aspiration and sclerotherapy for cystic diseases (1-4). Bleomycin-induced pulmonary fibrosis is characterized by the remodeling of fibrotic tissue and collagen deposition, which primarily results from proliferation of aberrant fibroblasts and trans-differentiation to myofibroblasts $(28,31)$. This process results from alveolar epithelial injury and the recruitment and activation of immune and/or inflammatory cells, as well as the production and release of cytokines and chemokines. These inflammatory cells and substances induce the activation of fibroblasts and myofibroblasts to result in the activation of transforming growth factor- $\beta 1$ and the deposition of an excessively stiff and biochemically abnormal extracellular matrix (32). In intratracheal bleomycin models of pulmonary fibrosis, fibrosis development occurs from day 14 onward, and worsens until disease resolution or animal sacrifice (28). The results of the 
present study showed that the collagen fibers were abundant at day 7 , then gradually decreased and eventually disappeared from 14 to 84 days. The different types of inflammatory cells contribute to the activation of fibroblasts and myofibroblasts and the deposition of collagen, and activated T-lymphocytes, B-lymphocytes and macrophages likely collaborate to orchestrate post-injury tissue remodeling and fibrosis (32). The present study also revealed that collagen fibrous proliferation is preceded by inflammatory cell infiltration, with the latter lasting longer throughout the experiment.

The late epithelial regeneration by a single layer of flat cells after chemical ablation of the gallbladder is like that reported in animal experiments with different sclerosants (25-27). In animal models of bleomycin-induced pulmonary fibrosis, alveolar epithelial regeneration is hypothesized to an important repair process following lung injury, which is regulated by various cellular and physiological mechanisms (34). In the present study, the regenerating epithelium consisted of a single layer of flat cells that were different from the original columnar epithelium of the gallbladder, and thus did not possess secretory function. Therefore, it is reasonable that epithelial regeneration after bleomycin sclerotherapy is the repair response to bleomycin-induced epithelial injury.

The present study has some limitations. First, it had few early observation time points, so early or ultra-early histopathological changes, such as the epithelial injury and inflammatory processes, may have been undetected. Second, the molecular and cellular mechanisms of bleomycin sclerotherapy for cystic diseases were not investigated; thus, the mechanisms of bleomycin-induced pulmonary and dermal fibrosis may be different from those of bleomycin sclerotherapy for cystic diseases. Finally, the gallbladder is a hypervascular structure, particularly when compared with simple hepatic cysts, with thick muscular layers beneath the epithelium, which may result in substantial differences in the complex inflammatory processes.

In conclusion, the present study described a rabbit model of simple hepatic cyst, established by ligating the cholecystic duct and preserving cholecystic vessels. The time course of histopathological changes occurring after bleomycin sclerotherapy consisted of an epithelial destruction phase, inflammatory infiltration phase, collagenous proliferation phase and epithelial regeneration phase. Information derived from this model may help in understanding the cellular mechanisms involved in the sclerosis induced by intracyst bleomycin injection of hepatic cysts and the time course of their resolution. Furthermore, these findings suggest that the pathogenesis of complications after percutaneous bleomycin sclerotherapy for treatment of cystic diseases is related to the bleomycin-induced inflammatory reaction. The optimal follow-up time of the therapeutic outcome assessment should be after inflammation has been resolved.

\section{Acknowledgements}

Not applicable.

\section{Funding}

The present study was funded by the Medical Scientific Research Foundation of Guangdong Province of China (grant no. A2015530).

\section{Availability of data and materials}

The datasets used and/or analyzed during the present study are available from the corresponding author on reasonable request.

\section{Authors' contributions}

LL and YHL conceived and designed the study. LL, YHL and $\mathrm{NZ}$ acquired, analyzed and interpreted the data, and wrote and revised the manuscript. LL and YHL performed the statistical analysis. All authors have read and approved the final manuscript. LL and YHL confirm the authenticity of all the raw data.

\section{Ethics approval and consent to participate}

The present study was approved by the Biomedical Ethics Committee of Animal Experiments of Guangdong Medical Laboratory Animal Center (Guangdong, China; approval no. B201610-5). An Accreditation Certificate from the China National Accreditation Service for Conformity Assessment has been granted to this facility. All experimental procedures were performed in accordance with the Guide for the Care and Use of Laboratory Animals (National Institutes of Health). Every effort was made to minimize animal suffering and to use only the number of animals necessary for the acquisition of reliable data.

\section{Patient consent for publication}

Not applicable.

\section{Competing interests}

The authors declare that they have no competing interests.

\section{References}

1. Dietrich CF, Chiorean L, Potthoff A, Ignee A, Cui X and Sparchez Z: Percutaneous sclerotherapy of liver and renal cysts, comments on the EFSUMB guidelines. Z Gastroenterol 54: 155-166, 2016.

2. Wijnands TF, Görtjes AP, Gevers TJ, Jenniskens SF, Kool LJ Potthoff A, Ronot M and Drenth JP: Efficacy and safety of aspiration sclerotherapy of simple hepatic cysts: A systematic review. AJR Am J Roentgenol 208: 201-207, 2017.

3. Lam SC and Yuen HK: Medical and sclerosing agents in the treatment of orbital lymphatic malformations: What's new? Curr Opin Ophthalmol 30: 380-385, 2019.

4. Eissa A, El Sherbiny A, Martorana E, Pirola GM, Puliatti S, Scialpi M, Micali S, Rocco B, Liatsikos E, Breda A, et al; European Section of Uro-Technology (ESUT): Non-conservative management of simple renal cysts in adults: A comprehensive review of literature. Minerva Urol Nefrol 70: 179-192, 2018.

5. Paladine W, Cunningham TJ, Sponzo R, Donavan M, Olson K and Horton J: Intracavitary bleomycin in the management of malignant effusions. Cancer 38: 1903-1908, 1976.

6. Yura J, Hashimoto T, Tsuruga N and Shibata K: Bleomycin treatment for cystic hygroma in children. Nippon Geka Hokan 46: 607-614, 1977

7. Takahashi H, Nakazawa S and Shimura T: Evaluation of postoperative intratumoral injection of bleomycin for craniopharyngioma in children. J Neurosurg 62: 120-127, 1985.

8. Johnston SR, Adam A, Allison DJ, Smith P and Ind PW: Recurrent respiratory obstruction from a mediastinal bronchogenic cyst. Thorax 47: 660-662, 1992.

9. Li L, Chen CC and Zeng XQ: One-year results of single-session sclerotherapy with bleomycin in simple renal cysts. J Vasc Interv Radiol 23: 1651-1656, 2012. 
10. Souftas VD, Kosmidou M, Karanikas M, Souftas D, Menexes G and Prassopoulos P: Symptomatic abdominal simple cysts: Is percutaneous sclerotherapy with hypertonic saline and bleomycin a treatment option? Gastroenterol Res Pract 2015: 489363, 2015.

11. Li L, Zeng XQ and Li YH: CT-guided percutaneous large-needle aspiration and bleomycin sclerotherapy for bronchogenic cyst: Report of four cases. J Vasc Interv Radiol 21: 1045-1049, 2010.

12. Della Latta V, Cecchettini A, Del Ry S and Morales MA: Bleomycin in the setting of lung fibrosis induction: From biological mechanisms to counteractions. Pharmacol Res 97 122-130, 2015

13. Temmerman F, Chen F, Libbrecht L, Vander Elst I, Windmolders P, Feng Y,Ni Y,De Smedt H, Nevens F and van Pelt J: Everolimus halts hepatic cystogenesis in a rodent model of polycystic-liver-disease. World J Gastroenterol 23: 5499-5507, 2017.

14. Yu Y, Shumway KL, Matheson JS, Edwards ME, Kline TL and Lyons LA: Kidney and cystic volume imaging for disease presentation and progression in the cat autosomal dominant polycystic kidney disease large animal model. BMC Nephrol 20: 259, 2019

15. Divyashree S, Janhavi P, Ravindra PV and Muthukumar SP. Experimental models of polycystic ovary syndrome: An update. Life Sci 237: 116911, 2019.

16. Atilgan R, Ozkan ZS, Kuloglu T, Kocaman N, Baspinar M, Can B, Şimşek M and Sapmaz E: Impact of intracystic ethanol instillation on ovarian cyst diameter and adjacent ovarian tissue. Eur J Obstet Gynecol Reprod Biol 174: 133-136, 2014.

17. National Research Council (US): Committee for the Update of the Guide for the Care and Use of Laboratory Animals: Guide for the Care and Use of Laboratory Animals. 8th edition. National Academies Press, Washington, DC, pp1-154, 2011.

18. Feldman AT and Wolfe D: Tissue processing and hematoxylin and eosin staining. Methods Mol Biol 1180: 31-43, 2014.

19. Goldner J: A modification of the masson trichrome technique for routine laboratory purposes. Am J Pathol 14: 237-243, 1938.

20. Rehg JE, Bush D and Ward JM: The utility of immunohistochemistry for the identification of hematopoietic and lymphoid cells in normal tissues and interpretation of proliferative and inflammatory lesions of mice and rats. Toxicol Pathol 40: 345-374, 2012.

21. Taylor CR and Rudbeck L (eds): Dako Education Guide: Immunohistochemical Staining Methods. 6th edition. ppl-110, 2013

22. Schmidt MJ, Tschoeke A, Noronha L, Moraes RS, Mesquita RA, Grégio AM, Alanis LR, Ignácio SA, Santos JN, Lima AA, et al: Histochemical analysis of collagen fibers in giant cell fibroma and inflammatory fibrous hyperplasia. Acta Histochem 118 : $451-455,2016$
23. Varghese F, Bukhari AB, Malhotra R and De A: IHC Profiler: An open source plugin for the quantitative evaluation and automated scoring of immunohistochemistry images of human tissue samples. PLoS One 9: e96801, 2014.

24. Terada T, Nakanuma Y, Ohta T, Nagakawa T, Motoo Y, Harada A, Hamato $\mathrm{N}$ and Inaba T: Mucin-histochemical and immunohistochemical profiles of epithelial cells of several types of hepatic cysts. Virchows Arch A Pathol Anat Histopathol 419: 499-504, 1991.

25. Uchiyama N, Stridbeck H and Stenram U: Chemical sclerosis of the gallbladder. An experimental study in pigs of the effect of absolute ethanol and polidocanol on gallbladder epithelium. Acta Radiol 30: 427-431, 1989.

26. Aagaard BD, Wetter LA, Montgomery CK and Gordon RL: Heat ablation of the normal gallbladder in pigs. J Vasc Interv Radiol 5: 331-339, 1994.

27. Lee JH, Won JH, Bae JI, Kim JH, Lee HS and Jung SM: Chemical ablation of the gallbladder with acetic acid. J Vasc Interv Radiol 20: 1471-1476, 2009.

28. Williamson JD, Sadofsky LR and Hart SP: The pathogenesis of bleomycin-induced lung injury in animals and its applicability to human idiopathic pulmonary fibrosis. Exp Lung Res 41: 57-73, 2015.

29. Liu T, De Los Santos FG and Phan SH: The bleomycin model of pulmonary fibrosis. Methods Mol Biol 1627: 27-42, 2017.

30. Yamamoto T: Intradermal injections of bleomycin to model skin fibrosis. Methods Mol Biol 1627: 43-47, 2017

31. Hadjicharalambous MR and Lindsay MA: Idiopathic pulmonary fibrosis: Pathogenesis and the emerging role of long non-coding RNAs. Int J Mol Sci 21: 524, 2020.

32. Heukels P, Moor CC, von der Thüsen JH, Wijsenbeek MS and Kool M: Inflammation and immunity in IPF pathogenesis and treatment. Respir Med 147: 79-91, 2019

33. MacIntosh PW, Yoon MK and Fay A: Complications of intralesional bleomycin in the treatment of orbital lymphatic malformations. Semin Ophthalmol 29: 450-455, 2014.

34. Basil MC, Katzen J, Engler AE, Guo M, Herriges MJ, Kathiriya JJ, Windmueller R, Ysasi AB,Zacharias WJ, Chapman HA, et al: The cellular and physiological basis for lung repair and regeneration: Past, present, and future. Cell Stem Cell 26: 482-502, 2020. International (CC BY-NC-ND 4.0) License. 